Minireview

\title{
MicroRNA and vascular smooth muscle cell phenotype: new therapy for atherosclerosis?
}

\author{
Chunxiang Zhang
}

Address: RNA and Cardiovascular Research Laboratory, Department of Anesthesiology, New Jersey Medical School, University of Medicine and Dentistry of New Jersey, Newark, NJ 07101, USA. Email: zhangc3@umdnj.edu

\begin{abstract}
MicroRNAs (miRNAs) represent a class of small, non-coding RNAs that negatively regulate gene expression via degradation or translational inhibition of their target mRNAs. Recent studies have identified that miR-145 is the most abundant miRNA in normal arteries and vascular smooth muscle cells (VSMCs), and its expression is significantly downregulated in dedifferentiated VSMCs and atherosclerotic arteries. miR-145 plays a critical role in modulating VSMC phenotype. Because phenotypic modulation of VSMCs is an initial cellular event in the development of atherosclerosis, miRNAs, and miR-145 in particular, may represent new therapeutic targets for atherosclerosis.
\end{abstract}

\section{Introduction}

It is well established that the transition of vascular smooth muscle cells (VSMCs) from a differentiated phenotype to a dedifferentiated state plays a critical role in the pathogenesis of atherosclerosis [1]. The phenotypic modulation in VSMCs is accompanied by accelerated migration, proliferation and production of extracellular matrix components. Eventually, these cellular events result in the formation of atherosclerotic lesions. However, the molecular mechanisms involved in phenotypic control are still unclear.

MicroRNAs (miRNAs) are a class of endogenous, small, non-coding RNAs that can pair with sites in $3^{\prime}$ untranslated regions in mRNAs of protein-coding genes to downregulate their expression [2]. More importantly, one miRNA is able to regulate the expression of multiple genes because it can bind to its mRNA targets as either an imperfect or a perfect complement. Thus, a miRNA can be functionally as important as a transcription factor [3]. As a group, miRNAs may directly regulate at least $30 \%$ of the genes in a cell [4]. It is therefore not surprising that miRNAs are involved in the regulation of all major cellular functions. Recently, the role of miRNAs in cardiac cell differentiation has been described [5]. In this study, Kwon et al. demonstrated that miR-1 plays important roles in modulating cardiogenesis and in maintenance of muscle-gene expression by targeting transcripts encoding the Notch ligand Delta. Failure of progenitor cell differentiation to cardiac cells was found in some miR-1 mutants [5]. The biological roles of miR-145 in diverse cancer cells have been recently identified (reviewed in [6]). In a recent article reported by Xu et al. [7], miR-145 was found to be a critical switch for embryonic stem cell differentiation by repressing some core pluripotency factors.

Whether miRNAs participate in the phenotypic control of VSMCs was unknown until recently. In this respect, three independent groups have reported exciting new discoveries regarding the critical role of the VSMC-enriched miRNA, miR-145, in VSMC phenotypic modulation [8-10]. They identified that miR-145 plays a role not only in the differentiation of multipotent neural crest stem cells into VSMCs, but also in the differentiation of adult VSMCs. Other miRNAs that may participate in the phenotypic modulation of VSMCs are miR-143 and miR-221 [9-12]. This minireview summarizes the current research progress regarding the roles of miR-145 in the VSMC phenotype and the potential therapeutic opportunities of miRNAs in atherosclerotic vascular disease.

\section{miR-145 expression is downregulated in injured arteries and in atherosclerotic arteries} Ji et al. [13] demonstrated that miR-145 is the most abundant miRNA in arteries. Its expression is significantly downregulated in rat balloon-injured arteries with neointimal lesion growth $[8,13]$. A recent Nature article by Cordes et al. [9] showed that miR-145 expression was also decreased in mouse carotid arteries after ligation injury. More interestingly, transcripts of miR-145 were downregulated to nearly undetectable levels in atherosclerotic lesions containing neointimal hyperplasia [9]. Our own unpublished data also revealed that miR-145 is largely downregulated in atherosclerotic mouse and human arteries, although the downregulation is less pronounced compared with that from Cordes' study. One possible reason for the discrepancy may be related to tissue selection. It is clear that the expression of miR-145 is mainly localized in VSMCs [8]. If the selected atherosclerotic tissue had fewer VSMCs, the expression level of miR-145 could be lower.

miRNA, microRNA; miR-145, microRNA-145; PDGF, platelet-derived growth factor; VSMC, vascular smooth muscle cell. 


\section{miR-145 expression is decreased in differentiated VSMCs}

Recently, Cheng et al. [8] found that miR-145 is the most abundant miRNA in differentiated VSMCs. Also, its expression is quickly downregulated in subcultured dedifferentiated VSMCs and in dedifferentiated VSMCs induced by stimulation with platelet-derived growth factor (PDGF). Our unpublished data also indicate that miR-145 expression in VSMCs isolated from balloon-injured rat carotid arteries and atherosclerotic ApoE-knockout mouse aortas is significantly decreased compared with that in VSMCs isolated from normal control arteries. Cordes et al. [9] found that, during the development of arteries, the expression of miR-145 is associated with the state of VSMC differentiation. miR-145 expression is notably absent in the aorta and pulmonary arteries during later cardiogenesis, during which VSMCs and arteries are developing. In contrast, high transcript levels of miR-145 in VSMCs of the arteries are demonstrated postnatally, after VSMCs and arteries have completed their development [9].

\section{miR-145 is a critical modulator of VSMC phenotype}

Cordes et al. [9] demonstrated that miR-145 was necessary and sufficient to induce differentiation of multipotent neural crest stem cells into VSMCs. In addition, Cheng et $a l$. [8] identified for the first time that miR-145 is a critical modulator for the VSMC phenotype both in vitro and in vivo. VSMC differentiation marker genes such as $S M$ $\alpha$-actin, calponin, and $S M-M H C$ were downregulated by miR-145 downregulation, and upregulated by miR-145 upregulation. The regulatory effect of miR-145 on the VSMC phenotype was further verified by Cordes and colleagues [9]. In contrast, another co-expressed miRNA, miR-143, had no significant effect on marker genes for VSMC differentiation, although it had a strong effect on VSMC proliferation. Boettger et al. [10] demonstrated that the expression of the miR-143/145 cluster is confined to VSMCs during development. They found that miR-143 and miR-145 are required for VSMC acquisition of the contractile phenotype because the VSMCs from miR-143/145deficient mice are locked in the synthetic state. The regulatory effects of miRNAs on the VSMC phenotype are not limited to miR-143 and miR-145. Two recent studies revealed that miR-221 is also related to the VSMC phenotype that affects VSMC proliferation and migration $[11,12]$.

\section{Do miRNAs represent new therapeutic targets for atherosclerosis?}

It is well known that phenotypic modulation of VSMCs is an initial cellular event in the development of atherosclerotic vascular disease. To determine the therapeutic potential of miR-145 in vascular disease, two recent studies have been reported $[8,10]$. Cheng et al. $[8]$ demonstrated that restoration of miR-145 in rat balloon-injured carotid arteries via adenovirus-mediated gene transfer significantly

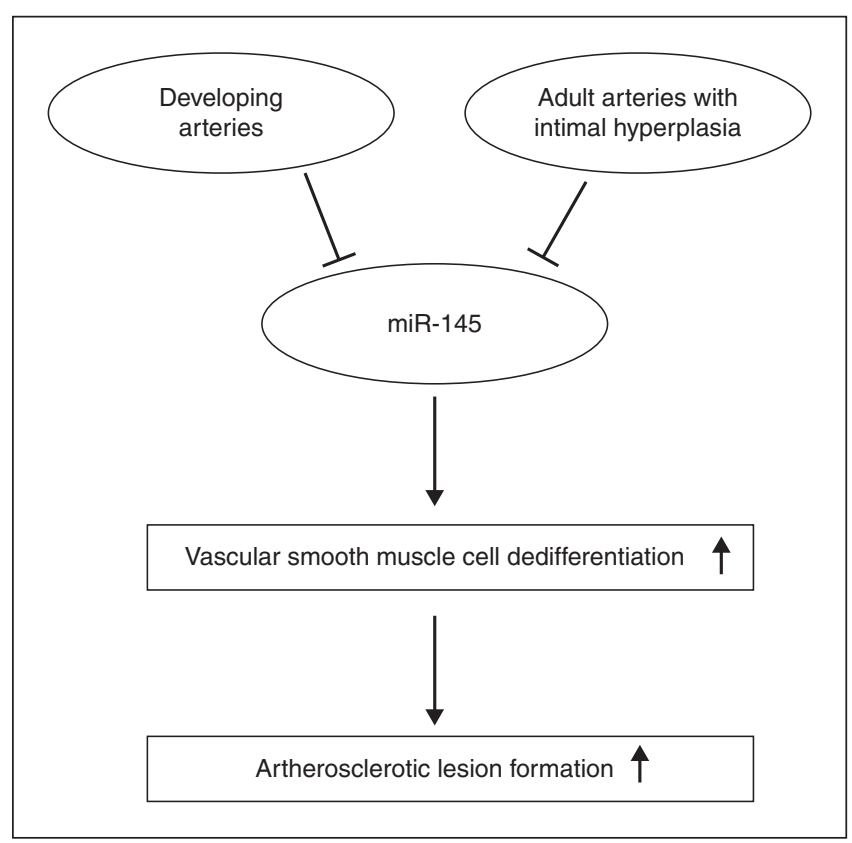

Figure 1

Expression modulation of miR-145 in the vascular walls and the potential roles of miR-145 in vascular smooth muscle cell phenotype and atherosclerosis. miR-145 is the most abundant miRNA in normal arteries. Its expression is significantly downregulated in developing arteries and in adult arteries containing intimal hyperplasia. The downregulated miR-145 in adult arteries will increase VSMC dedifferentiation and result in the development of atherosclerotic lesions.

inhibited neointimal lesion growth. In contrast, knockout of miR-143 and miR-145 resulted in the formation of neointima in mouse arteries [10]. The roles of miR-145 in the VSMC phenotype and atherosclerotic vascular disease are summarized in Figure 1.

Atherosclerotic vascular disease is a multifactorial complex disease in which many genes are involved. miRNA-based therapy may have some advantages compared with that for other molecular targets because one endogenous miRNA can target its multiple target genes. Although the recent studies have demonstrated that targeting miRNAs, and miR-145 in particular, may represent a new therapy for atherosclerosis, there is still a long road before miRNAbased technology can be translated to clinical therapy. First, the critical miRNAs responsible for the development of atherosclerosis must be further identified, especially in human atherosclerotic arteries. Second, the detailed cellular and molecular mechanisms of these critical miRNAs in the prevention and treatment of atherosclerosis should be studied. Third, in addition to the biological effects of these miRNAs on VSMCs, their effects on other atherosclerosisrelated cellular events should be identified. Fourth, although methods are available to downregulate a miRNA 
in vivo, technology for upregulating a miRNA in the vascular walls in vivo requires development. Finally, the potential side-effects of miRNA-based therapy should be studied before application in the clinic.

\section{Competing interests}

The author declares that he has no competing interests.

\section{Acknowledgements}

This work was supported by a National Institutes of Health Grant HL080133 and a grant from the American Heart Association 09GRNT2250567.

\section{References}

1. Owens GK, Kumar MS, Wamhoff BR: Molecular regulation of vascular smooth muscle cell differentiation in development and disease. Physiol Rev 2004, 84:767-801.

2. Ambros V: The functions of animal microRNAs. Nature 2004; 431:350-355.

3. Chen K, Rajewsky N: The evolution of gene regulation by transcription factors and microRNAs. Nat Rev Genet 2007, 8:93-103.

4. Lewis BP, Burge CB, Bartel DP: Conserved seed pairing, often flanked by adenosines, indicates that thousands of human genes are microRNA targets. Cell 2005, 120:15-20.

5. Kwon C, Han Z, Olson EN, Srivastava D: MicroRNA1 influences cardiac differentiation in Drosophila and regulates Notch signaling. Proc Natl Acad Sci USA 2005, 102:1898618991.

6. Wang Y, Lee CG: MicroRNA and cancer - focus on apoptosis. J Cell Mol Med 2009, 13:12-23.
7. Xu N, Papagiannakopoulos T, Pan G, Thomson JA, Kosik KS: MicroRNA-145 regulates OCT4, SOX2, and KLF4 and represses pluripotency in human embryonic stem cells. Cell 2009, 137:647-658.

8. Cheng $Y$, Liu X, Yang J, Lin Y, Xu D, Lu Q, Deitch EA, Huo $Y$, Delphin E, Zhang C: MicroRNA-145, a novel smooth muscle cell phenotypic marker and modulator, controls vascular neointimal lesion formation. Circ Res 2009, 105:158-166.

9. Cordes KR, Sheehy NT, White MP, Berry EC, Morton SU, Muth AN, Lee TH, Miano JM, Ivey KN, Srivastava D: miR-145 and miR-143 regulate smooth muscle cell fate and plasticity. Nature 2009, 460:705-710.

10. Boettger T, Beetz N, Kostin S, Schneider J, Krüger M, Hein L, Braun T: Acquisition of the contractile phenotype by murine arterial smooth muscle cells depends on the Mir143/145 gene cluster. J Clin Invest, 2009, 119:2634-2647.

11. Davis BN, Hilyard AC, Nguyen PH, Lagna G, Hata A: Induction of microRNA-221 by platelet-derived growth factor signaling is critical for modulation of vascular smooth muscle phenotype. J Biol Chem 2009, 284:37283738 .

12. Liu X, Cheng $Y$, Zhang S, Lin Y, Yang J, Zhang C: A necessary role of miR-222 and miR-221 in vascular smooth muscle cell proliferation and neointimal hyperplasia. Circ Res 2009, 104:476-487.

13. Ji R, Cheng Y, Yue J, Yang J, Liu X, Chen H, Dean DB, Zhang C: MicroRNA expression signature and antisense-mediated depletion reveal an essential role of microRNA in vascular neointimal lesion formation. Circ Res 2007, 100: 1579-1588.

Published: 9 September 2009

doi:10.1186/gm85

(c) 2009 BioMed Central Ltd 